\section{PET and SPECT in Psychiatry}

R.A.J.O. Diercks, A. Otte, E.F.J. de Vries, and A. van Waarde, eds.

New York, NY: Springer, 2014, 849 pages, $\$ 399.00$

This book aims to introduce PET and SPECT studies while helping readers better understand the gist of psychiatric disorders. Because PET and SPECT provide information on neurochemical transmission as well as on brain perfusion and metabolism, they have historically had a crucial role in disclosing neurobiology and developing psychiatric drugs. This book helped us grasp the current status of psychiatric neuroimaging studies and the future direction of their clinical use.

The first part of the book presents the basics of neuroimaging in psychiatry. The role of PET in psychiatric drug development is introduced, and microdosing pharmacokinetics, receptor occupancy, and imaging biomarkers are explained. PET microdosing for new-radioligand development was recently introduced and requires a profound understanding of tracer kinetics and PET quantification ranging from compartment modeling of bolus infusions to neurotransmitter level monitoring and comparison studies with microdialysis. The book explains the importance of measuring neurochemicals that can be manipulated by pharmacologic and neurologic interventions. The book's presentation of the story of P-glycoprotein is another merit because of the role P-glycoprotein has had in slowing the development of brain radiochemicals.

Parts 2-6 present the use of PET and SPECT studies for specific psychiatric disorders. Part 2 includes comprehensive reviews of neuroimaging for depressive disorders, categorized as studies of metabolism/blood flow, neurochemical serotonin systems, dopamine and monoamine oxidase, and depression, including late-life depression, suicide attempts, and seasonal depression. There is also a focus on the use of PET and SPECT in such psychiatric problems as depression observed in dementia disorders and Parkinson disease. Part 3 introduces anxiety disorders. The chapters summarize well the alteration in neurotransmission while explaining the pharmacologic and pathophysiologic background.

The remainder of the book presents PET and SPECT findings in psychosis, particularly schizophrenia and delirium. Too few studies are described to elucidate any pathophysiologic implications. Nevertheless, despite the small number of studies, these chapters may still benefit the reader. The PET and SPECT studies on personality disorders are comprehensive, and the section on the use of PET and SPECT in drug addiction covers both preclinical and clinical studies as well as the neurobiologic background of addiction. In addition, in the same chapter one can find a clinical overview of impulsivity and a description of how we can image impulsivity by targeting neurotransmission. For obsessive readers such as us who reach the final pages of the book, the last chapter is a great joy. It explains the significant changes that can occur in neuroimages due to alterations in brain metabolism and neurochemicals

COPYRIGHT (c) 2015 by the Society of Nuclear Medicine and Molecular Imaging, Inc. brought about by such interventions as neurostimulation, behavior therapy, and surgical treatment.

The contents of several chapters overlap. A detailed review of the neuroimaging of behavioral and psychologic symptoms of dementia would have improved the book, as would an initially presented general description of dementia. PET studies should have been better explained, considering their clinical importance in schizophrenia, including their use to define endophenotypes.

Studying the neurobiology and pathophysiology of psychiatric disorders is difficult because of their complexity and uniqueness. This book will certainly provide readers with a chance to better understand how functional neuroimaging studies can be used to answer fundamental questions about psychiatric diseases.

Hongyoon Choi
Dong Soo Lee*
*Seoul National University College of Medicine
28 Yungundong Chonnogu
Seoul 110-744, Korea
E-mail: dsl@plaza.snu.ac.kr

Published online Aug. 20, 2015.

DOI: 10.2967/jnumed.115.164608

\section{PET and SPECT in Neurology}

R.A.J.O. Diercks, A. Otte, E.F.J. de Vries, and A. van Waarde, eds.

New York, NY: Springer, 2014, 1,112 pages, $\$ 399$

PET and SPECT in Neurology consists of 2 major sections. The first (part 1) covers the basics of using PET and SPECT to describe brain function and deals with tracer kinetics and quantification methods. In anticipation of the potential future clinical use of PET/MR imaging in neurology, the book also presents the use of MR techniques to examine blood flow, metabolism, and neuronal transmission. One can even find a description of the default-mode brain network on resting-state functional MR imaging, as well as interpretations in terms of the functional, structural, and metabolic networks.

In the second section of the book (parts 2-8), the clinical application and current status of using PET and SPECT imaging in several neurologic disorders are described, including dementia, cerebrovascular disorders, movement disorders, inflammation, epilepsy, and brain tumors. The clinical application of ${ }^{18}$ F-FDG and amyloid PET in dementia, and of dopamine transporter SPECT in movement disorders, are reviewed excellently in textbook fashion. The current knowledge on these topics is nothing new, but the information is strongly recommended for beginners in PET and SPECT who want to thoroughly and systematically learn the present status of these imaging techniques in neurology.

Besides covering PET and SPECT findings for Alzheimer disease and mild cognitive impairment, the book appropriately emphasizes PET and SPECT findings for vascular dementia. Associated MR 